\title{
MINIMAL TIME CONTROL OF PNEUMATIC CYLINDER AND ITS PERFORMANCE
}

\author{
Osamu OYAMA* and Toshihiro YOSHIMITSU* \\ * Department of Precision Engineering, School of Science and Technology, \\ Meiji University \\ 1-1-1, Higashimita, Tama-ku,Kawasaki,214-8751 Japan
}

\begin{abstract}
A pneumatic cylinder is usually used for the positioning of point to point and the switching timing of control valve have been tuned experimentally. To reduce the operating time or/and consumption energy for control of cylinder, the switching timing of valve must be tuned taking account the obvious index of performance. The switching process derived from the index is analyzed and the performances such as operating time, consumption energy and accuracy of the control are shown comparing the minimal time control and minimal energy control, in this report.
\end{abstract}

\section{KEYWORDS}

Optimal control, Pneumatic servo, Pneumatic cylinder, Minimal time control, Minimal energy control

\section{INTRODUCTION}

Many study about a control of pneumatic cylinder deal the dynamics of control system[1][2][3][4][5][6] [7]. This report deals a minimizing control method on operation time or consumption energy of pneumatic cylinder which is fed air with on-off type solenoid valve. In generally, to analyze the optimal control condition such as minimal operation time or/and minimal consumption energy control conditions, the maximum principle is well used. This principle has a merit which can deduce the control low in the system having the limitation of control parameters such as saturation of flow rate of control air in solenoid valve, although this theory needs to solve the differential equation to meet many boundary conditions and then it can not be easily solved. In the other hand, a displacement of pneumatic cylinder in rather long range has a terminated velocity caused by a saturation of control flow and increase of frictional force according to the increase of velocity, and its characteristic is suitable for solving the equation by reducing the boundary condition about velocity versus position.

This report deals the positioning control of pneumatic cylinder at desired position freely selected in stroke. The minimal time control which minimize the time for completing the positioning of pistoncylinder is realized as follows. The piston starts for desired position by supplying air from the control onoff valve, and when it reaches at proper position ahead of desired position, the valve discharges the air of cylinder to reduce a velocity of piston quickly and the valve shuts off to keep balance at the desired position finally. On the other hand, the minimal energy control is realized by shutting off the supply valve before cylinder reaches to the desired position[8]. In this report, the meaning of 'consumption energy' represents the total air flow volume needed to complete the positioning and 'minimal energy' means also the minimum supply air volume which can settle the cylinder at desired position. In both controls, the 
follow conditions must be realized as a result of the control when the cylinder reaches at desired position. The velocity becomes zero and the cylinder pressure comes to balance against the load weight. If each or both conditions are not realized, piston can not settle at this position, so these conditions are treated as the end side boundary conditions.

Furthermore, we show the features of minimal time control derived from maximum principle comparing with the minimal energy control and an improvement method on positioning accuracy of these control by applying an added control stage of switching operation of the solenoid valve is proposed.

\section{EXPERIMENTAL SETUP}

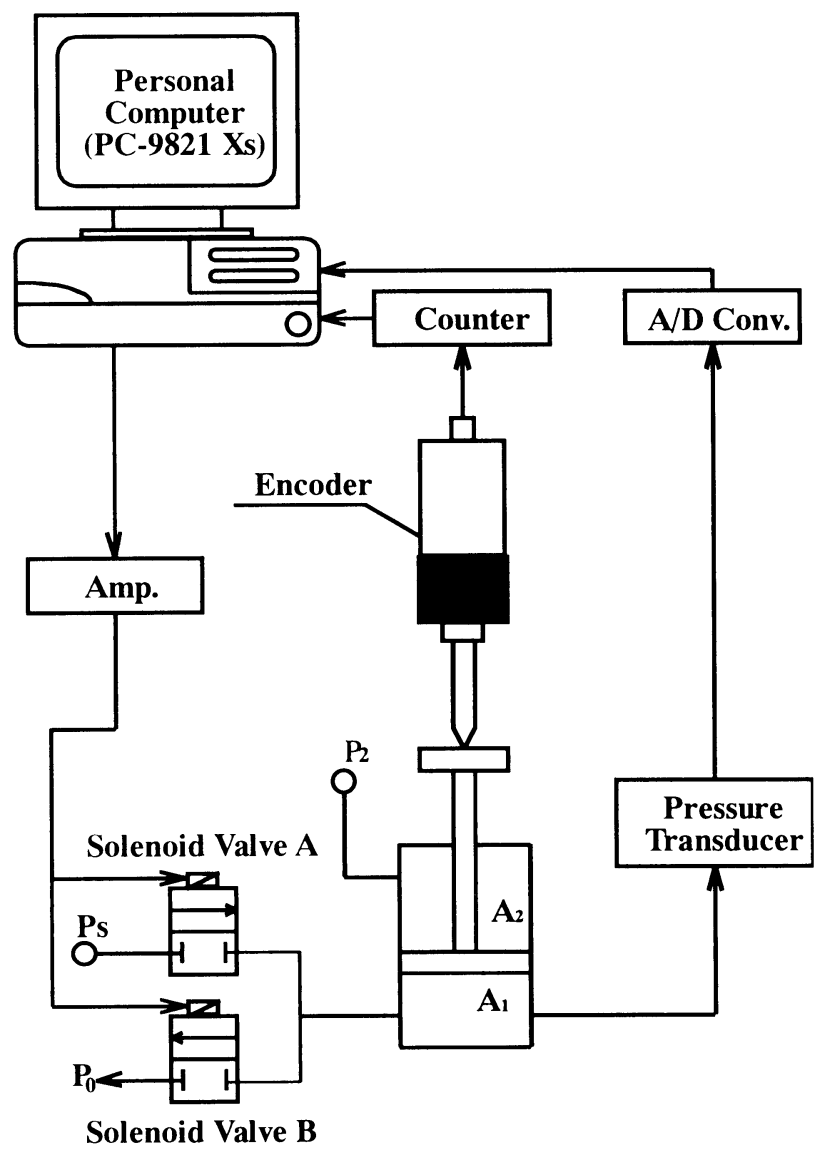

Figure 1 Scheme of control device

The experimental scheme of pneumatic cylinder positioning system is shown in Fig. 1. A single rod type cylinder is used locating vertical direction and is yielded a constant pressure to rod side. The stroke is $100 \mathrm{~mm}$ and bore is $40 \mathrm{~mm}$, respectively. Two on-off type solenoid valves are connected to head side of cylinder. The valve A supplies the air and the valve $\mathrm{B}$ discharges the air to ambient. The supply pressure of valve is $0.5 \mathrm{MPa}$. Only one of the valves opens according to the control low of minimal time or minimal energy. The displacement of piston is measured by linear scale encoder of which resolution is $0.001 \mathrm{~mm}$ and the pressure in the head side cylinder is measured by pressure sensor and these values are used for control. A load weight is $7.8 \mathrm{~N}$. The flow meter is set at the exhaust port of valve $B$ to measure the total air volume needed in each positioning, although it is not shown in this figure.

\section{THEORY}

The nomenclatures used in this chapter are as follows.

$P 1$ : Pressure in the head side cylinder

$A 1$ :Cross-sectional area of head side piston

[Pa]

$A 2$ :Cross-sectional area of rod side piston

$$
\begin{array}{ll}
M: \text { Mass of Piston } & {[\mathrm{kg}]} \\
D: \text { Viscous frictional force } & {[\mathrm{N}]} \\
C: \text { Capacitance of cylinder } & {[\mathrm{m} / \mathrm{Pa}]} \\
F c: \text { Coulomb frictional force } & {[\mathrm{N}]} \\
R: \text { Flow resistance of solenoid valve } & {\left[\mathrm{Pas} / \mathrm{m}^{3}\right]}
\end{array}
$$

\subsection{Minimal time control}

The minimal time control can be done by keeping to supply the maximum flow rate with valve $A$ until piston reaches at proper position ahead of final desired position and then discharging the air of cylinder by opening valve $\mathrm{B}$ in order to decrease the velocity of piston. And when the piston reaches at desired position the valves shut off, at this time, velocity becomes zero and the pressure reaches the balanced pressure against load weight. The first position where valve $\mathrm{A}$ closes and Valve $\mathrm{B}$ opens is very important to succeed the control and to know the position according to various conditions, theoretical analyze by using the maximum principle is done. 
As the performance index $\mathrm{J}$ for minimal time control is selected as follow.

$$
J=\int_{t l}^{t 2} 1 d t=t 2-t 1
$$

Where, $t 1$ is a start time and $t 2$ is a end time when positioning is completed. The state functions of pneumatic servo system are as follows

$\dot{x}_{0}=1$

$\dot{x}_{1}=x_{2}$

$\dot{x}_{2}=-\frac{D}{M} x_{2}+\frac{A_{1}}{M} x_{3}-\frac{1}{M}\left(P_{2} A_{2}+F_{c}+M g\right)$

$\dot{x}_{3}=-\frac{A_{1}}{C} x_{2}-\frac{1}{C R} x_{3}+\frac{P_{s}}{C R} u$

Using vector $\phi=\left(\begin{array}{llll}\phi_{0} & \emptyset_{1} & \phi_{2} & \phi_{3}\end{array}\right)^{T}$, Hamilton function $\mathrm{H}$ are defined as follows.

$H=\emptyset_{0}+\phi_{1} x_{2}+\phi_{2}\left\{-\frac{D}{M} x_{2}+\frac{A_{1}}{M} x_{3}-\frac{1}{M}\left(P_{2} A_{2}+F_{c}+M g\right)\right\}$

$+\emptyset_{3}\left(-\frac{A_{1}}{C} x_{2}-\frac{1}{C R} x_{3}+\frac{P_{s}}{C R} u\right)$

As input of system $u, u=1$ (if valve $A$ opens) and $u=0$ (if Valve $\mathrm{B}$ opens) are defined. A vector $\varphi=\left(\begin{array}{llll}\phi_{0} & \phi_{1} & \phi_{2} & \phi_{3}\end{array}\right)^{T}$ must meet the conditions as follows.

$\dot{\phi}_{0}=0$

$\dot{\phi}_{1}=0$

$\dot{\phi}_{2}=-\phi_{1}+\frac{D}{M} \phi_{2}+\frac{A_{1}}{C} \phi_{3}$

$\dot{\phi}_{3}=-\frac{A_{1}}{M} \phi_{2}+\frac{1}{C R} \phi_{3}$

The term about input $\mathrm{u}$ in the hamilton function is

$H(u)=\phi_{3} \frac{P_{s}}{C R} u$

and the control low which minimizes equ.(1) is derived from the condition which yields the maximum value as the $\mathrm{H}(\mathrm{u})$, i.e.,

if $\Psi_{3} \geqq 0$ then $\mathrm{u}=1$, and if $\Psi_{3}<0$ then $\mathrm{u}=0$

The on-off operations of valve $A$ and $B$ are derived from equ.(6) by calculating the $\Psi_{3}$ using the measured initial values of position and pressure. The calculation is executed by off line before the control.

The both boundary conditions which must be satisfied between start and end of control are as Table
1. Equ.(2) and (4) must be solved to meet these conditions and it is very difficult and if one of these conditions is negligible the calculation becomes easy dramatically. In generally, a velocity of piston has terminated value when its displacement is rather long and this characteristics is suitable to reduce the conditions. The reduced boundary conditions are shown in Table 2. The equations are solved from $t 2$ to $\mathrm{t} 1$ inversely as actual time and the start time $\mathrm{t} 1$ is decided from the calculation which meets the start conditions.

Table 1 Boundary conditions

\begin{tabular}{|c|c|c|}
\hline & start & end \\
\hline time & $\mathrm{t} 1$ & $\mathrm{t} 2$ \\
\hline position & initial value & desired \\
\hline velocity & zero & zero \\
\hline pressure & initial value & yielded by load \\
\hline
\end{tabular}

Table 2 Reduced boundary conditions

\begin{tabular}{|c|c|c|}
\hline & start & end \\
\hline time & $\mathrm{t} 1$ & $\mathrm{t} 2$ \\
\hline position & free & desired \\
\hline velocity & $\begin{array}{c}\text { terminated } \\
\text { value }\end{array}$ & zero \\
\hline pressure & supply pressure & yielded by load \\
\hline
\end{tabular}

The valve operation is done when the piston reaches beforehand decided position by calculation as shown before and this operation is open loop control fundamentally and actual valve on-off position which satisfies such conditions is effected by various disturbances, then the position derived from theory is tuned experimentally in practical test.

\subsection{Minimal energy control}

In ordinary valve operation of positioning about point to point such as stroke-ends, the supply control valve keeps to open and additional air comes after the positioning, so unnecessary air consumption may occur. From this point of view, Kawakami and others have 
proposed the method which shuts off the valve before the piston reaches at the desired position(7). In this report, to define the minimal energy condition more clearly, the positioning at desired position ahead of stroke-end which is yielded obvious boundary conditions as same as minimal time control is selected. In this case, the additional condition differ from positioning at stroke-end is that the cylinder pressure must agree the balanced value to load weight when piston reaches desired position.

The minimum energy control corresponds to the valve operation which minimizes the performance index J, i.e.,

$J=\int_{t 1}^{t^{2}} G(t) d t$

where $G(t)$ is flow rate. Though the operation of valve may be derived from maximum principle as same as minimal time control, the opening duration of valve $\mathrm{A}$ is tuned to meet the boundary conditions experimentally, because it is clear that the valve $\mathrm{B}$ does not open in this case.

\section{EXPERIMENTAL RESULTS}

\subsection{Comparison of features in minimal time and minimal energy control}

The enlarged wave form of piston displacement near the settling position is shown in Fig.2 comparing

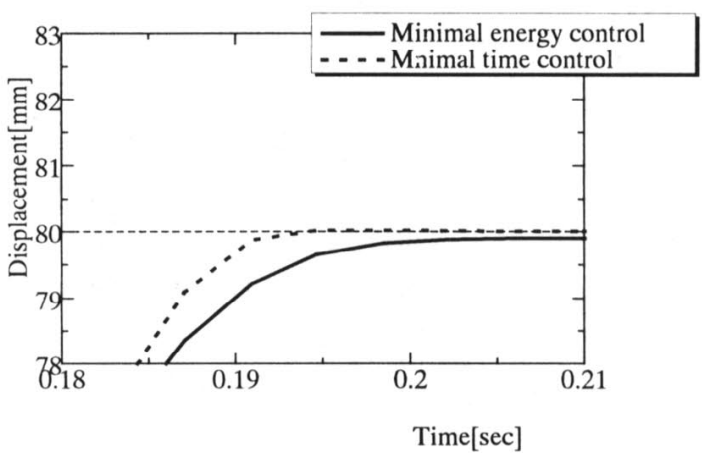

Figure 2 Piston displacement

the case of minimal time control and minimal energy control. A desired position is $80 \mathrm{~mm}$ point from lower stroke-end and the start position is this stroke- end, although the wave forms of piston displacement near the start are not shown in this figure. The dotted line denotes the case of minimal time control and the piston settles quickly at desired position than the other.

The comparison of settling time from start is shown in Table 3. The value of minimal time control is smaller than that of minimal energy control although the difference is not so large, because the displacement of piston is not long and the load weight is not so large in this case, and the difference of settling time becomes larger as increase of them.

Table 3 Comparison of settling time

\begin{tabular}{|c|c|}
\hline Minimal time control & minimal energy control \\
\hline $0.216 \mathrm{~s}$ & $0.243 \mathrm{~s}$ \\
\hline
\end{tabular}

The consumption energy of positioning is evaluated as the amount of supplied air volume on the once positioning by measuring the sum of the discharged air through the valve $\mathrm{B}$ during positioning and the exhausted air thorough this valve when the piston returns back to initial position after the positioning is completed. The air volume is shown as normal condition in the ambient.

The minimal time control requires to discharge the air in order to decrease the piston velocity rapidly near the desired position, on the other hand, the minimal energy control does not discharge the air during positioning and then the consumption air volume of later control is smaller comparing with the minimal time control. To specify the increment of consumption air volume of minimal time control, the comparison of volume needed on once positioning in the both controls is done. Table 4(a) shows the consumption air volume of each control. Although the air volume, in other words, the energy in minimal time control is larger than that of minimal energy control naturally, the difference is very small because the discharged air in minimal time control before complete the positioning is very small and is almost 2 percent of total air volume. Then it is shown that the minimal time control is not so inferior in energy saving, 
and is expected to shorten the needed time of pneumatic cylinder works, although the difference of energy consumption of both control may increases according to increase of load. Table 4(b) shows the theoretical values of air volume in both control. They fairly agree to experimental values.

Table 4 Consumption air volume

(a) Experiment $\left(10^{-4} \mathrm{~m}^{3}\right)$

\begin{tabular}{|c|c|c|}
\hline & Discharge air & Total air \\
\hline Minimal time & 0.07 & 4.18 \\
\hline Minimal energy & 0 & 4.08 \\
\hline
\end{tabular}

(b) Calculation $\left(10^{-4} \mathrm{~m}^{3}\right)$

\begin{tabular}{|c|c|c|}
\hline & Discharge air & Total air \\
\hline Minimal time & 0.09 & 4.23 \\
\hline Minimal energy & 0 & 4.15 \\
\hline
\end{tabular}

\subsection{Improvement of positioning accuracy}

Minimal time and minimal energy controls shown above are open loop control fundamentally so the positioning accuracy at the desired position is not well caused by disturbance or uncertain friction of cylinder. As the examples, the histograms of positioning result in the repeated tests of 50 times against $80 \mathrm{~mm}$ desired point are shown in Figure 3 and Figure 4. The former shows the result of minimal time control and the later shows that of minimal energy control, respectively. All data come in the range of plus/minas $0.15 \mathrm{~mm}$ but it is not negligible. The main reason of occurring the deviation is that the cylinder displacement during the valve opens is long and the control error tends to pile up.

To improve the accuracy, two stage control is proposed. The first stage is coarse displacement to near the desired point and the second stage is short displacement to correct the final error, in later stage, the valve operation is simple action of short duration.. The first desired position of this stage that is tentative is 90 percent of final desired position and as soon as the cylinder settles here, the measurement of position and pressure is done and the control parameter that is an opening duration of valve $\mathrm{A}$ are decided from these values. The second stage displacement is short and the final position error becomes small naturally. If the second stage starts ahead of settling of piston on the first step, the measured values are not proper for the next control and as a result, the cylinder fails to overshoot the desired position. To ensure this settling duration, the measurements of position and pressure is delayed, i.e., the start of second step is also delayed. The Figure 5 shows the displacement of cylinder near the desired position of $80 \mathrm{~mm}$ when the delay time varies at $10 \mathrm{~ms}$ interval from zero to $50 \mathrm{~ms}$. It is found that the delay of $30 \mathrm{~ms}$ is proper to avoid a overshoot across the desired position and it is a waste of time to increase the delay more, in this case.

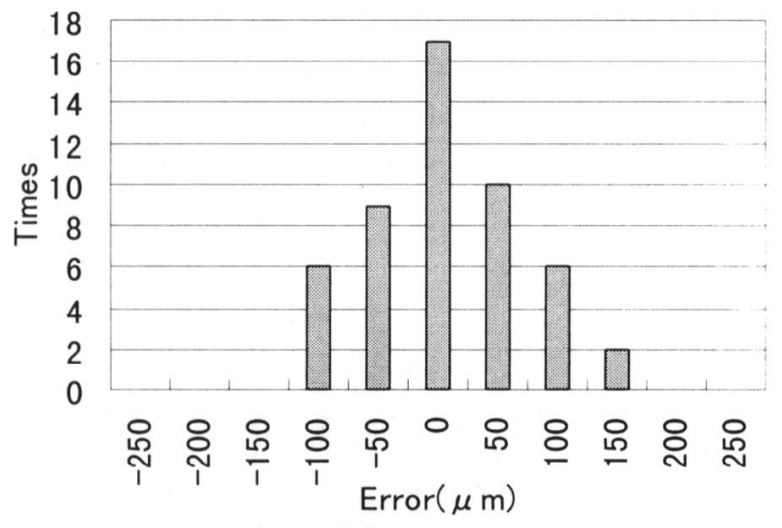

Figure 3 Positioning accuracy on minimal time control

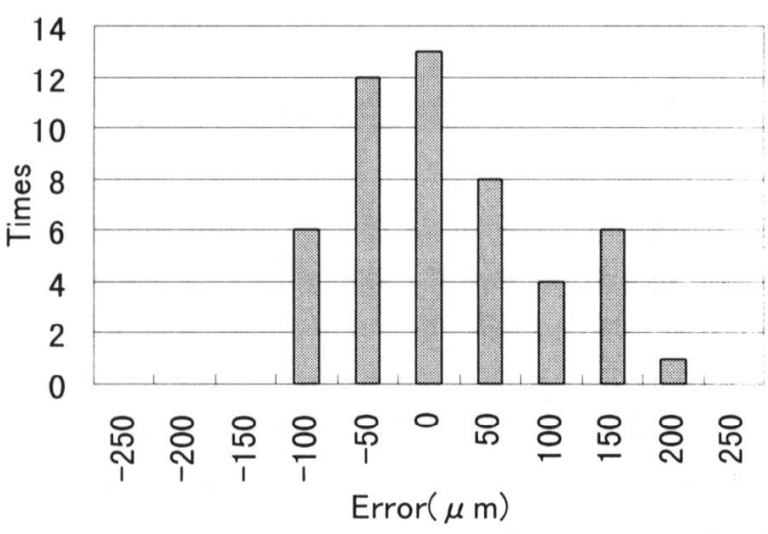

Figure 4 Positioning accuracy on minimal energy control

The comparisons of positioning accuracy are shown as distribution of errors in Figure 6. The black 
bars show the result of ordinary control and the gray bars show that of two step control described above, respectively. The positioning error is improved clearly when two step control is done.

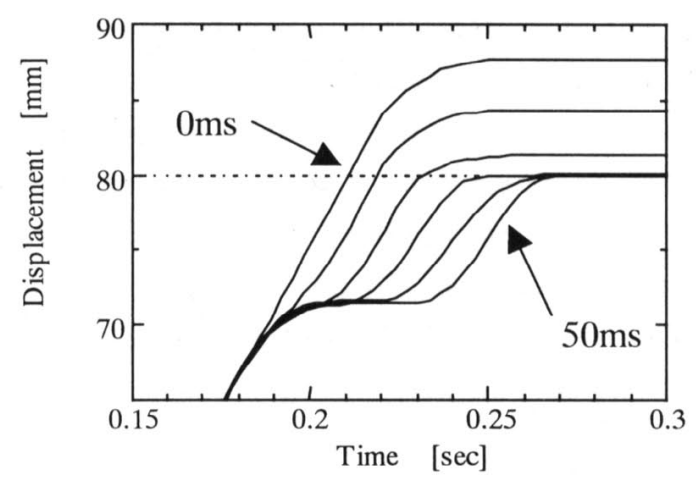

Figure 5 Effect of time delay on piston displacement

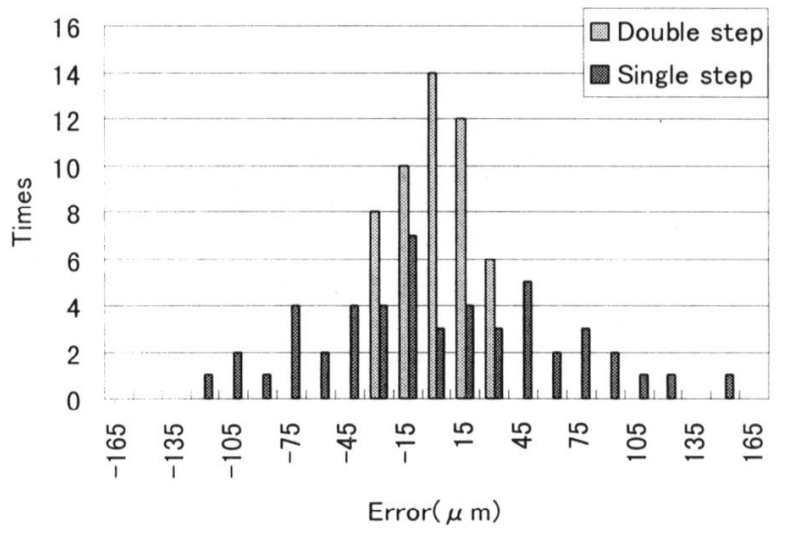

Figure 6 Comparison of positioning accuracy

\section{CONCLUSION}

The efficiencies of various works are needed to improve and the control of pneumatic cylinder must be done by using obvious performance index. In this report, minimal time control derived from performance index of operation time of cylinder work is proposed and its feature is shown comparing with the minimal energy control derived from the point of view of minimizing consumption air flow. As a result, it is specified that the operation time of minimal time control can be shorten clearly and the increase of consumption air is not so much.

\section{ACKNOWLEDGMENT}

The authors would like to express gratitude to SMC Corp. for offers of equipment.

\section{REFERENCES}

1.. Araki, K., Yin, Y.B. and OChen, J.B., High Speed Force Control of a Pneumatic Asymmetric Valve-Controlled Cylinder with a Fuzzy Controller, Proceedings of Fifth Triennial International Symposium on Fluid Control, Measurement and Visualization, 1997, pp.485-495

2. Noritsugu, T. and Fukuzuno, K., FORCE CONTROL OF PNEUMATIC SERVO SYSTEM USING NEURAL NETWORK, Proceedings of the Third JHPS International Symposium on Fluid Power'96, 1996, pp.139-144

3. Araki, K., Suzuki, K. and Osada, T., THE FORCE CONTROL OF A SPOT WELDING MACHINE WITH A SPECIALLY DESIGNED PNEUMATIC CYLINDER, Proceedings of the Third JHPS International Symposium on Fluid Power'96, 1996, pp.343-348

4. Virvalo, T., COMPARING DIFFERENT CONTROLLERS OF ELECTROPNEUMATIC POSITION SERVO, Proceedings of the Third JHPS International Symposium on Fluid Power'96, 1996, pp.151-156

5. Kawanaka, H. and Hanada, K., MANY POINTS POSITIONING CONTROL OF A PNEUMATIC CYLINDER FOR A VERTICAL AXIS ACTUATOR USING TWO DEGREES OF FREEDOM PI CONTROLLER, Proceedings of the Third JHPS International Symposium on Fluid Power'96, 1996, pp.157-161

5. Oyama, O., Preview Control of Pneumatic Servo Cylinder, Proceedings of Fifth Triennial International Symposium on Fluid Control, Measurement and Visualization, 1997, pp.265270

6. Horiguchi, K., Shinozaki, K., Kawakami, Y., Terashima, Y. and Kawai, S., Study on a pneumatic Cylinder System with Energy-Saving, Proceedings of Fifth Triennial International Symposium on Fluid Control, Measurement and Visualization, 1997, pp.479-484 